\title{
A KöMaL problem in a new view
}

\author{
PÁl PÉter DÁlyay
}

\begin{abstract}
The object of this paper is finding the general solution $f: \mathbb{R}^{3} \rightarrow \mathbb{R}$ of the system of functional equations (1) valid for all $x, y, z, t \in \mathbb{R}$. First $f$ is expressed by a function of one variable which satisfies a system of two functional equations. This system is resolved by using an algebraic reformulation of the problem in terms of orbits and transversals. Finally the general solution of (1) is obtained.
\end{abstract}

Key words and phrases: general solution of a system of functional equations, transformation group, orbit, transversal.

ZDM Subject Classification: Primary I70, Secondary H40.

1. The problem and solvers' questions

The solution of problem B.3438. was published in No. 7./ October 2001 of mathematical and physical journal for secondary schools KöMaL. We refresh the text of the problem:

B.3438. The function $f(x, y, z)$ satisfies

$$
\left\{\begin{array}{l}
f(x+t, y+t, z+t)=t+f(x, y, z) \\
f(t x, t y, t z)=t \cdot f(x, y, z) \\
f(x, y, z)=f(y, x, z)=f(x, z, y)
\end{array}\right.
$$

for every real number $t$. Compute $f(2000,2001,2002)$. 
The editorial comment about the solutions remarks that some solvers found the function $f(x, y, z)=\frac{x+y+z}{3}$ and one of them found an entire family the functions of which satisfy equations (1), these functions are:

$$
f_{a}(x, y, z)= \begin{cases}\frac{x+y+z}{3} & \text { if } x \neq y \neq z \neq x \\ x+a \cdot(y-x) & \text { if } y=z \\ y+a \cdot(x-y) & \text { if } x=z \\ z+a \cdot(x-z) & \text { if } x=y\end{cases}
$$

where $a$ is an arbitrary real number.

So the following question may be raised: What is the set of the solutions of system of functional equations (1)?

We propose to find all functions $f: \mathbb{R}^{3} \rightarrow \mathbb{R}$ which satisfy the system (1).

\section{A function of one variable} instead of one of three variables

We are going to find all functions $f: \mathbb{R}^{3} \rightarrow \mathbb{R}$ which satisfy the system of functional equations (1) for all $x, y, z, t \in \mathbb{R}$. For this purpose we will use the following theorem.

Theorem 1. A function $f: \mathbb{R}^{3} \rightarrow \mathbb{R}$ satisfies the system of functional equations (1) for all $x, y, z, t \in \mathbb{R}$ if and only if there is a solution $g: \mathbb{R} \rightarrow \mathbb{R}$ of the system of functional equations

$$
\begin{cases}g(t)+g(1-t)=1 & \text { if } t \in \mathbb{R} \\ g(t)=t \cdot g\left(\frac{1}{t}\right) & \text { if } t \in \mathbb{R} \backslash\{0\}\end{cases}
$$

which satisfies the following equality

$$
f(x, y, z)= \begin{cases}x+(y-x) \cdot g\left(\frac{z-x}{y-x}\right) & \text { if } \quad x \neq y, x, y, z \in \mathbb{R} \\ x+(z-x) \cdot g(0) & \text { if } \quad x=y, x, y, z \in \mathbb{R}\end{cases}
$$


Proof. Let $f$ be a function satisfying system (1) for all $x, y, z, t \in \mathbb{R}$. Equations of (1) imply

$$
\begin{aligned}
f(x, y, z)= & x+f(0, y-x, z-x) \\
& = \begin{cases}x+(y-x) \cdot f\left(0,1, \frac{z-x}{y-x}\right) & \text { if } x \neq y \\
x+(z-x) \cdot f(0,0,1) & \text { if } x=y\end{cases} \\
& = \begin{cases}x+(y-x) \cdot f\left(0,1, \frac{z-x}{y-x}\right) & \text { if } x \neq y \\
x+(z-x) \cdot f(0,1,0) & \text { if } x=y\end{cases}
\end{aligned}
$$

If we set $g(t)=f(0,1, t)$ for all $t \in \mathbb{R}$ then equality (3) is a direct consequence of previous relations.

On the basis of the third equation of system (1) the equation $f(x, y, z)=$ $f(y, x, z)$ holds. Using $(3)$ for $x \neq y$ it follows that

$$
x+(y-x) \cdot g\left(\frac{z-x}{y-x}\right)=y+(x-y) \cdot g\left(\frac{z-y}{x-y}\right) .
$$

If we use the notation $t=\frac{z-x}{y-x}$ then $1-t=\frac{z-y}{x-y}$ and from the previous relation yields

$$
g(t)+g(1-t)=1 \quad \text { if } t \in \mathbb{R}
$$

If $y \neq x \neq z$ then equations $f(x, y, z)=f(x, z, y)$ and (3) imply

$$
x+(y-x) \cdot g\left(\frac{z-x}{y-x}\right)=x+(z-x) \cdot g\left(\frac{y-x}{z-x}\right)
$$

and according to the previous notations we have

$$
g(t)=t \cdot g\left(\frac{1}{t}\right) \quad \text { if } t \in \mathbb{R} \backslash\{0\} .
$$

We have shown that if a function $f: \mathbb{R}^{3} \rightarrow \mathbb{R}$ satisfies the system of functional equations (1) for all $x, y, z, t \in \mathbb{R}$ then there is a function $g: \mathbb{R} \rightarrow \mathbb{R}$, defined by $g(t)=f(0,1, t)$ for all $t \in \mathbb{R}$, which satisfies system (2) and equality (3). So the direct part have proved.

Conversely, we assume that $g: \mathbb{R} \rightarrow \mathbb{R}$ is a solution of system of functional equations (2) and $f: \mathbb{R}^{3} \rightarrow \mathbb{R}$ is a function satisfying equation (3) and we will prove that $f$ satisfies system (1) for all $x, y, z, t \in \mathbb{R}$. 
On the basis of (3) it is obvious that the first two equations of (1) are satisfied.

The equality $f(x, y, z)=f(y, x, z)$ for $x=y$ is trivial. For $x \neq y$ it results from the first equation of system (2). If we put $t=\frac{z-x}{y-x}$ in (2) and multiply it by $y-x$ then we obtain

$$
(y-x) \cdot g\left(\frac{z-x}{y-x}\right)+(y-x) \cdot g\left(\frac{z-y}{x-y}\right)=y-x
$$

which is equivalent to $f(x, y, z)=f(y, x, z)$.

To prove equality $f(x, y, z)=f(x, z, y)$ we will examine the following cases:

$1^{\circ}$ If $x=y$ and $x \neq z$ then according to $(3)$ we have $f(x, y, z)=x+(z-x) \cdot g(0)$ and $f(x, z, y)=x+(z-x) \cdot g\left(\frac{y-x}{z-x}\right)=x+(z-x) \cdot g(0)$ and our equality holds. We may conclude that $f(x, x, z)=f(x, z, x)$ for all $x, z \in \mathbb{R}$.

$2^{\circ}$ If $x \neq y$ and $x=z$ then using the previous equality we obtain: $f(x, y, z)=$ $f(x, y, x)=f(x, x, y)=f(x, z, y)$.

$3^{\circ}$ If $x \neq y$ and $x \neq z$ then $f(x, y, z)=x+(y-x) \cdot g\left(\frac{z-x}{y-x}\right)$ and $f(x, z, y)=$ $x+(z-x) \cdot g\left(\frac{y-x}{z-x}\right)$. In this case our equality is a direct consequence of the second equation of system (2) by substitution $t=\frac{z-x}{y-x}$. According to the previous verifications we may conclude that equation $f(x, y, z)=f(x, z, y)$ holds for every $x, y, z \in \mathbb{R}$. So the converse is also true.

We remark that the problem of finding the functions of three variables satisfying system (1) can be reduced to the problem of finding the functions of one variable satisfying system of functional equations (2). We have already shown that a solution $f: \mathbb{R}^{3} \rightarrow \mathbb{R}$ of (1) determines a solution $g: \mathbb{R} \rightarrow \mathbb{R}$ of (2) by $g(t)=f(0,1, t)$ for all $t \in \mathbb{R}$. If we denote by $\mathcal{H}_{1}$ the set of solutions of system (1) and by $\mathcal{H}_{2}$ the set of solutions of (2) then we can introduce a map $\Phi: \mathcal{H}_{1} \rightarrow \mathcal{H}_{2}$ by $\Phi(f)=g$, where $g(t)=f(0,1, t)$ for all $t \in \mathbb{R}$. The converse part of Theorem 1 shows that every solution $g: \mathbb{R} \rightarrow \mathbb{R}$ of system (2) also determines a solution of system (1) by formula (3). In consequence we can define a map $\Psi: \mathcal{H}_{2} \rightarrow \mathcal{H}_{1}$ by formula $\Psi(g)=f$, where $f$ is the function given by formula (3). It is easy to verify that for every $f \in \mathcal{H}_{1}$ and for every $g \in \mathcal{H}_{2}$ we have $\Psi(\Phi(f))=f$, and $\Phi(\Psi(g))=g$. These relations mean that $\Phi$ and $\Psi$ are the inverses of each other. We may conclude that every function of $\mathcal{H}_{1}$ can be obtained from a function of $\mathcal{H}_{2}$ and inversely.

Before we begin to resolve system (2) it is advisable to make a halt in algebra. We are going to mention some algebraic notions which are useful to make more clearly the process of solving of the system. 


\section{Transformation groups, orbits and transversals}

The notion of group is fundamental among algebraic structures. Important examples of groups are transformation groups. A map from a set $X$ into itself is called transformation of the set $X$. In the set of transformations of $X$ we introduce the composition of the maps as operation, which will be denoted by $\circ$. A transformation of the set $X$ is called permutation of $X$ if it is a bijection. We denote by $S(X)$ the set of all permutations of $X$. It is clear that the composite of two permutations is a permutation. For more information about introduced notions consult texts [2] and [3].

Example 1. The set of all permutations of a set $X$ with operation the composition of the maps form a group, i.e. $(S(X), \circ)$ is group. Indeed, the composition of the maps is associative. The identity map of $X$ denoted by $\varepsilon$ is a permutation of $X$ and for every $f \in S(X)$ we have $\varepsilon \circ f=f \circ \varepsilon=f$, i.e. $\varepsilon$ is the identity of $S(X)$ relative to the composition of the maps. Finally, if $f \in S(X)$, then $f$ has an inverse $f^{-1}$ such that $f^{-1} \in S(X)$ and $f \circ f^{-1}=f^{-1} \circ f=\varepsilon$, i.e. for every element of $S(X)$ there is an inverse in $S(X)$.

A group $G$ is called a transformation group if there is a set $X$ such that $G$ is a subgroup of $(S(X), \circ)$.

Example 2. Let $X=R \backslash\{0,1\}$. Let $\varepsilon, \sigma, \theta, \alpha, \beta$ and $\gamma$ be the following permutations of $X: \varepsilon: x \mapsto x, \sigma: x \mapsto 1-x, \theta: x \mapsto \frac{1}{x}, \alpha: x \mapsto \frac{x-1}{x}, \beta: x \mapsto$ $\frac{x}{x-1}, \gamma: x \mapsto \frac{1}{1-x}$. For these permutations we have $\sigma \circ \sigma: x \mapsto 1-\sigma(x)=x$, $\theta \circ \theta: x \mapsto \frac{1}{\theta(x)}=x, \sigma \circ \theta: x \mapsto 1-\frac{1}{x}=\frac{x-1}{x}$, i.e. $\sigma \circ \sigma=\varepsilon, \theta \circ \theta=\varepsilon$ and $\sigma \circ \theta=\alpha$. Analogously, $\theta \circ \alpha=\beta, \sigma \circ \beta=\gamma$ and $\theta \circ \gamma=\sigma$. It follows $\alpha=\sigma \circ \theta, \beta=\theta \circ(\sigma \circ \theta)$, $\gamma=\sigma \circ(\theta \circ(\sigma \circ \theta))$ and $\sigma=\theta \circ(\sigma \circ(\theta \circ$ $(\sigma \circ \theta)))$. These calculations show that the set $G=\{\varepsilon, \sigma, \theta, \alpha, \beta, \gamma\}$ is closed relative to composition of permutations, and every element of $G$ has its inverse in $G$. This means that the subgroup generated by the set $\{\sigma, \theta\}$ in the group $(S(X), \circ)$ is exactly $G=\{\varepsilon, \sigma, \theta, \alpha, \beta, \gamma\}$. So $G$ is a transformation group. The operation table of this

\begin{tabular}{|c|cccccc|}
\hline$\circ$ & $\varepsilon$ & $\sigma$ & $\theta$ & $\alpha$ & $\beta$ & $\gamma$ \\
\hline$\varepsilon$ & $\varepsilon$ & $\sigma$ & $\theta$ & $\alpha$ & $\beta$ & $\gamma$ \\
\hline$\sigma$ & $\sigma$ & $\varepsilon$ & $\alpha$ & $\theta$ & $\gamma$ & $\beta$ \\
\hline$\theta$ & $\theta$ & $\gamma$ & $\varepsilon$ & $\beta$ & $\alpha$ & $\sigma$ \\
\hline$\alpha$ & $\alpha$ & $\beta$ & $\sigma$ & $\gamma$ & $\theta$ & $\varepsilon$ \\
\hline$\beta$ & $\beta$ & $\alpha$ & $\gamma$ & $\sigma$ & $\varepsilon$ & $\theta$ \\
\hline$\gamma$ & $\gamma$ & $\theta$ & $\beta$ & $\varepsilon$ & $\sigma$ & $\alpha$ \\
\hline
\end{tabular}
group is: 
We assume now that $X$ is a set and $H$ is a group of transformations of $X$ relative to the operation of composition. We also assume that the identity element of the group is $\varepsilon$ the identity transformation of $X$. These conditions are fulfilled for both our examples. The orbit of an arbitrary element $x$ of $X$ relative to the transformation group $H$ is the set of all elements of $X$ which may be obtained as values of a transformation belonging to $H$ in $x$. So the orbit of $x$ is the set: $\widehat{x}=\{\eta(x) \mid \eta \in H\}$. Because $\varepsilon \in H$ we have $x \in \widehat{x}$.

Next we are examining the relation between two orbits. If the orbits $\widehat{x}$ and $\widehat{y}$ have a common element $z$, then there are two transformations $\eta_{1}$ and $\eta_{2}$ in $H$ such that $\eta_{1}(x)=z$ and $\eta_{2}(y)=z$. Because $H$ is a group the inverse of $\eta_{2}$ relative to the composition of maps $\eta_{2}^{-1}$ is also in $H$ so $\eta_{2}^{-1} \circ \eta_{1} \in H$. But $\left(\eta_{2}^{-1} \circ \eta_{1}\right)(x)=\eta_{2}^{-1}(z)=y$ implies $y \in \widehat{x}$. It follows $\widehat{y}=\{\xi(y) \mid \xi \in H\}=$ $\left\{\xi\left(\eta_{2}^{-1}\left(\eta_{1}(x)\right)\right) \mid \xi \in H\right\} \subseteq\{\zeta(x) \mid \zeta \in H\}=\widehat{x}$. If we change the role of $x$ and $y$ we obtain $\widehat{x} \subseteq \widehat{y}$ and conclude $\widehat{x}=\widehat{y}$.

We have proved that two orbits are equal or they are disjoint.

The concept of the transversal is related to the notion of orbit. A subset of $X$ is a transversal relative to a transformation group $H$ if it meets each orbit in a single point.

Which are the orbits and the transversals in our previous examples? In the first example there is a single orbit itself the set $X$, because for a pair of elements $x, y \in X$ there is a permutation of $X$ which maps $x$ to $y$. So every one point set is a transversal.

The second problem is more interesting. If $r$ is a real number different from 0 and 1 , then the orbit of $r$, the set $\widehat{r}=\{\xi(r) \mid \xi \in G\}$ contains at most six elements. Because every element of the set $X=\mathbb{R} \backslash\{0,1\}$ has an orbit we conclude that in this case there are infinitely many different orbits.

Now we are showing that the interval $[2,+\infty)$ is a transversal. The elements of $G$ are continuous and strictly monotonous functions on the interval $(2,+\infty)$. Using this fact we may determine the images of $(2,+\infty)$ produced by each element of $G$ :

$$
\begin{aligned}
& \sigma((2,+\infty))=(-\infty,-1), \quad \gamma((2,+\infty))=(-1,0), \quad \theta((2,+\infty))=\left(0, \frac{1}{2}\right) \\
& \alpha((2,+\infty))=\left(\frac{1}{2}, 1\right), \quad \beta((2,+\infty))=(1,2), \quad \varepsilon((2,+\infty))=(2,+\infty) .
\end{aligned}
$$


Because these intervals are disjoint and every orbit of an element of interval $(2,+\infty)$ has exactly one element in each of these image sets we conclude that the orbits of these elements have six elements. It is also obvious that every element of each of these image belongs to the orbit of some element of $(2,+\infty)$. The elements of $X$ don't belong to these image sets are $-1, \frac{1}{2}, 2$. It is easy to verify that they form an orbit with three elements, so $\widehat{2}=\left\{-1, \frac{1}{2}, 2\right\}$.

We have proved that the interval $[2,+\infty)$ is a transversal because each orbit contains exactly one element of this interval.

\section{Solving of system of functional equations (2)}

The general solution of system of functional equations (2) is given by the following theorem.

Theorem 2. A function $g: \mathbb{R} \rightarrow \mathbb{R}$ satisfies the system of functional equations (2) if and only if a function $h:[2,+\infty) \rightarrow \mathbb{R}$ with $h(2)=1$ and a real number $r$ exist such that they verify the relation

$$
g(t)= \begin{cases}\widetilde{h}(t) & \text { if } t \in \mathbb{R} \backslash\{0,1\} \\ 1-r & \text { if } t=0 \\ r & \text { if } t=1\end{cases}
$$

where function $\widetilde{h}$ is an extension of $h$ to the set $\mathbb{R} \backslash\{0,1\}$ by formula

$$
\widetilde{h}(t)= \begin{cases}1-h(1-t) & \text { if } t \in(-\infty,-1] \\ t \cdot\left[1-h\left(\frac{t-1}{t}\right)\right] & \text { if } t \in[-1,0) \\ t \cdot h\left(\frac{1}{t}\right) & \text { if } t \in\left(0, \frac{1}{2}\right] \\ 1+(t-1) \cdot h\left(\frac{1}{1-t}\right) & \text { if } t \in\left[\frac{1}{2}, 1\right) \\ t+(1-t) \cdot h\left(\frac{t}{t-1}\right) & \text { if } t \in(1,2] \\ h(t) & \text { if } t \in[2,+\infty) .\end{cases}
$$


Proof. Before we begin to prove this theorem we may mention that the double definitions of certain values of extension $\widetilde{h}$ are without contradictions, this fact is assured by the condition $h(2)=1$.

First we suppose that a function $g: \mathbb{R} \rightarrow \mathbb{R}$ satisfies system (2). Using the functions of group $G$ the equations of the system may be written in the following form:

$$
g(\sigma(t))=\sigma(g(t))=1-g(t)
$$

respectively:

$$
g(\theta(t))=\frac{g(t)}{t}
$$

Using the operation table of $G$ and the equations (6) and (7) we obtain:

$$
\begin{aligned}
& g(\alpha(t))=g(\sigma(\theta(t)))=\sigma(g(\theta(t)))=1-\frac{g(t)}{t} \\
& g(\beta(t))=g(\theta(\alpha(t)))=\frac{g(\alpha(t))}{\alpha(t)}=\frac{1-\frac{g(t)}{t}}{\frac{t-1}{t}}=\frac{t-g(t)}{t-1} \\
& g(\gamma(t))=g(\theta(\sigma(t)))=\frac{g(\sigma(t))}{\sigma(t)}=\frac{1-g(t)}{1-t}
\end{aligned}
$$

We define the function $h$ as the restriction of $g$ to the interval $[2,+\infty)$. Because $\beta(2)=2$ under (9) the condition $g(2)=1$ results, so we have $h(2)=1$. It is easy to check that we have the image sets:

$$
\begin{aligned}
& \sigma([2,+\infty))=(-\infty,-1] ; \quad \gamma([2,+\infty))=[-1,0) ; \quad \theta([2,+\infty))=\left(0, \frac{1}{2}\right] \\
& \alpha([2,+\infty))=\left[\frac{1}{2}, 1\right) ; \quad \beta([2,+\infty))=(1,2] ; \quad \varepsilon([2,+\infty))=[2,+\infty)
\end{aligned}
$$

The relations (6)-(11) show that if we give the value of function $g$ at a point $t \in[2,+\infty)$ then we may obtain the value of $g$ for each element of orbit of $t$. So restriction $h$ of $g$ to the interval $[2,+\infty)$ determines all the values of function $g$ on the set $X=\bigcup_{\tau \in G} \tau([2,+\infty))=\mathbb{R} \backslash\{0,1\}$. The values of $g$ at 0 and 1 are connected by relation (6). If the value of $g$ at 1 is $r$ then $g(0)=1-r$. According 
to these observations we have the relation:

$$
g(t)= \begin{cases}1-h\left(\sigma^{-1}(t)\right) & \text { if } t \in(-\infty,-1] \\ \frac{1-h\left(\gamma^{-1}(t)\right)}{1-\gamma^{-1}(t)} & \text { if } t \in[-1,0) \\ \frac{h\left(\theta^{-1}(t)\right)}{\theta^{-1}(t)} & \text { if } t \in\left(0, \frac{1}{2}\right] \\ \frac{h\left(\alpha^{-1}(t)\right)}{\alpha^{-1}(t)} & \text { if } t \in\left[\frac{1}{2}, 1\right) \\ \frac{\beta^{-1}(t)-h\left(\beta^{-1}(t)\right)}{\beta^{-1}(t)-1} & \text { if } t \in(1,2] \\ h(t) & \text { if } t \in[2,+\infty) \\ 1-r & \text { if } t=0 \\ r & \text { if } t=1 .\end{cases}
$$

After calculations it follows (4). We have proved that there are a function $h$ : $[2,+\infty) \rightarrow \mathbb{R}$ with $h(2)=1$ and a real number $r$ which satisfy relations (4) and (5). This means that the first part of the theorem is true.

Conversely, now we assume that a function $g$ is given by relations (4) and (5) for an arbitrary function $h:[2,+\infty) \rightarrow \mathbb{R}$ with $h(2)=1$ and for a real number $r$. These formulas show that functions $g$ and $h$ satisfy relation (12). We compute $g(\sigma(t))$. Using the relations $\sigma^{-1} \circ \sigma=\varepsilon, \gamma^{-1} \circ \sigma=\beta^{-1}, \theta^{-1} \circ \sigma=\alpha^{-1}$, $\alpha^{-1} \circ \sigma=\theta^{-1}, \beta^{-1} \circ \sigma=\gamma^{-1}$, and the following equivalences $\sigma(t) \in(-\infty,-1] \Longleftrightarrow$ $t \in[2,+\infty), \sigma(t) \in[-1,0) \Longleftrightarrow t \in(1,2], \sigma(t) \in\left(0, \frac{1}{2}\right] \Longleftrightarrow t \in\left[\frac{1}{2}, 1\right)$, $\sigma(t) \in\left[\frac{1}{2}, 1\right) \Longleftrightarrow t \in\left(0, \frac{1}{2}\right], \sigma(t) \in(1,2] \Longleftrightarrow t \in[-1,0), \sigma(t) \in[2,+\infty) \Longleftrightarrow$ $t \in(-\infty,-1]$ we obtain: 


$$
g(\sigma(t))= \begin{cases}h\left(\sigma^{-1}(t)\right) & \text { if } t \in(-\infty,-1] \\ \frac{\gamma^{-1}(t)-h\left(\gamma^{-1}(t)\right)}{\gamma^{-1}(t)-1} & \text { if } t \in[-1,0) \\ 1-\frac{h\left(\theta^{-1}(t)\right)}{\theta^{-1}(t)} & \text { if } t \in\left(0, \frac{1}{2}\right] \\ \frac{h\left(\alpha^{-1}(t)\right)}{\alpha^{-1}(t)} & \text { if } t \in\left[\frac{1}{2}, 1\right) \\ \frac{1-h\left(\beta^{-1}(t)\right)}{1-\beta^{-1}(t)} & \text { if } t \in(1,2] \\ 1-h(t) & \text { if } t \in[2,+\infty) \\ r & \text { if } t=0 \\ 1-r & \text { if } t=1 .\end{cases}
$$

On the basis of relations (12) and (13) results that (6) holds for all $t \in \mathbb{R}$. An analogous verification shows that $(7)$ holds for all $\in \mathbb{R} \backslash\{0\}$. So $g$ is a solution of system (2).

\section{A family of solutions}

Finally we present the family of solutions generated by the linear function $h:[2,+\infty) \rightarrow \mathbb{R}$ of the form $h(t)=p t+1-2 p$, where $p$ is an arbitrary real number. Firstly we determine the functions $g$ corresponding to function $h$ by formula (12):

$$
g(t)= \begin{cases}p \cdot t+p & \text { if } t \in(-\infty, 0) \\ 1-r & \text { if } t=0 \\ (1-2 p) \cdot t+p & \text { if } t \in(0,1) \\ r & \text { if } t=1 \\ p \cdot t+1-2 p & \text { if } t \in(1,+\infty),\end{cases}
$$


where $r$ is an arbitrary real number. According to Theorem 1 the corresponding solutions $f$ of system (1) may be obtained by formula (3):

$$
f(x, y, z)= \begin{cases}x+p \cdot(y+z-2 x) & \text { if } \frac{z-x}{y-x}<0 \\ y+r \cdot(x-y) & \text { if } z=x \\ z+p \cdot(x+y-2 z) & \text { if } 0<\frac{z-x}{y-x}<1 \\ x+r \cdot(y-x) & \text { if } y=z \\ y+p \cdot(x+z-2 y) & \text { if } \frac{z-x}{y-x}>1 \\ z+r \cdot(x-z) & \text { if } x=y .\end{cases}
$$

This family of solutions of system of functional equations (1) depends on two real parameters $p$ and $r$. If we put $r=1-p$ then obtain solutions of a simpler form:

$$
f(x, y, z)= \begin{cases}x+p \cdot(y+z-2 x) & \text { if }(z-x)(y-x) \leq 0 \\ z+p \cdot(x+y-2 z) & \text { if } 0<\frac{z-x}{y-x}<1 \\ y+p \cdot(x+z-2 y) & \text { if } \frac{z-x}{y-x} \geq 1\end{cases}
$$

If we put $p=\frac{1}{3}$ then from (16) obtain the function $f(x, y, z)=\frac{x+y+z}{3}$ and from (15) the family of functions mentioned in the first part of this article.

\section{References}

[1] Dályay Pál Péter, Még egyszer a B.3438. feladatról, KöMaL 52. évfolyam 3. szám, Budapest (2002 március).

[2] Nathan Jacobson, Lectures in Abstract Algebra, Springer-Verlag, New York, Heidelberg, Berlin, 1975.

[3] Szendrei Ágnes, Diszkrét matematika, Polygon jegyzetsorozat, Szeged, 1996.

DÁLYAY PÁL PÉTER

DEÁK FERENC HIGH SCHOOL

JÓZSEF ATTILA SGT. 118-120, 6723 SZEGED

HUNGARY

E-mail: dpp@freemail.hu

(Received March, 2003) 